\title{
Modulation of the renin-angiotensin system and retinopathy
}

\author{
Nish Chaturvedi
}

Diabetic retinopathy is still the leading cause of blindness in people of working age. It is one of the most common complications of diabetes, affecting $80-100 \%$ of type 1 patients and being present at the diagnosis of diabetes in around one third of type 2 patients.

\section{Pathology}

The earliest detectable lesions of diabetic retinopathy are microaneurysms. These are associated with excessive vascular permeability and result in exudate formation. There is occlusion of the vessels and stimulation of growth factors, which in turn promote the proliferation of new blood vessels and the growth of fibrous tissue. This may contract and lead to haemorrhage. The process of angiogenesis, however, occurs right through the evolution of diabetic retinopathy.

\section{Treatment}

Until very recently, treatment was confined to relatively late in the pathology of diabetic retinopathy, when there was already some proliferative disease. Photocoagulation has been successful in preventing blindness and, more recently, studies have shown the importance of tight blood glucose control.

GLYCAEMIC CONTROL

The diabetes control and complications trial (DCCT) in patients with type 1 diabetes emphasised the importance of tight glycaemic control in reducing the progression of retinopathy and also reducing the incidence of retinopathy (fig 1). ${ }^{1}$

Importantly, even patients receiving intensive treatment had some progression of retinopathy. Also, it took three years before a clear difference between intensive and conventional treatment was observed. Clearly, while tight glycaemic control is successful, other treat-

\footnotetext{
The EURODIAB Data Co-ordinating Centre, University College London, Department of Epidemiology and Public Health, 1-19 Torrington Place, London WC1E 6BA, UK

N Chaturvedi

Correspondence to: Dr Chaturvedi email: n.chaturvedi@ic.ac.uk
}

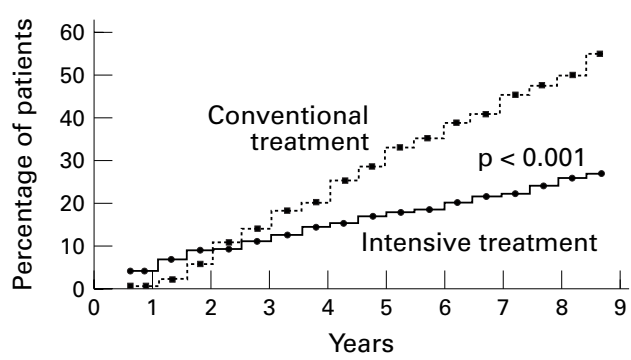

Figure 1 Results of DCCT showing the progression of retinopathy with conventional and intensive treatment to achieve glycaemic control. Reproduced from DCCT Research Group. N Engl f Med 1993;329:977-86. Copyright (C) 1993. Massachusetts Medical Society. All rights reserved. ments are still required. There is now strong evidence that agents that modulate the reninangiotensin system (RAS) may be one of these treatments.

\section{RAS and retinopathy}

A local RAS exists in the eye that is independent of the systemic RAS. There is now evidence that patients with diabetic retinopathy have raised concentrations of intraocular and serum angiotensin converting enzyme (ACE), prorenin, and angiotensin II. These concentrations are correlated with disease severity.

The non-haemodynamic effects of angiotensin II include the regulation of cell growth via the expression of growth factors including vascular endothelial growth factor; basic fibroblast growth factor; insulin-like growth factor; and platelet derived growth factor. Angiotensin II also enhances vascular permeability and oxidant stress; and potentiates sympathetic nervous system activity. It may also be involved in retinal vascular tone and the degradation of the extracellular matrix.

As angiotensin II has such a wide range of actions, it is not surprising that there is evidence that it is involved in the pathogenesis of diabetic retinopathy.

ANGIOTENSIN II AS AN ANGIOGENIC FACTOR In vitro studies have shown the impact of angiotensin II as an angiogenic factor. In cultured cells, angiotensin II potentiates cell migration. However, this effect is attenuated when either captopril or an $\mathrm{AT}_{1}$ receptor antagonist is added. ${ }^{2}$

A human study looked at the impact of ACE inhibition in the eye in nine patients who were followed for seven months. Captopril was found to improve significantly blood/retinal barrier permeability and albumin excretion rate $(\mathrm{p}<0.01)$. $^{3}$

UKPDS

In the UKPDS study a group of type 2 diabetic patients with hypertension were randomised to tight blood pressure control or less tight blood pressure control. ${ }^{4}$ After a period of three years there was an improvement in the progression of retinopathy in those randomised to tight blood pressure control. There was also an improvement in visual acuity.

UKPDS also compared ACE inhibitor with $\beta$ blocker treatment and for the majority of end points, including retinopathy, there was no distinction between the two agents. However, as this was a subgroup analysis the results should be interpreted with caution. A twice daily dosing schedule of captopril was used that may not 


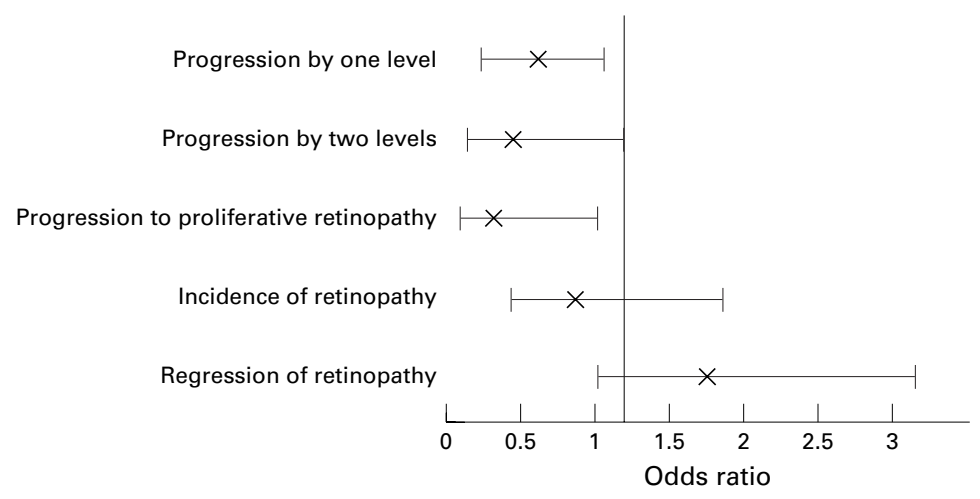

Figure 2 Odds ratios and $95 \%$ confidence intervals for the treatment effects of lisinopril in the EUCLID study. control and those with relatively poor glycaemic control. Those on treatment in either of these two groups had a better outcome than those on placebo, and there was no significant interaction between the two groups.

\section{Microalbuminuria}

The number of people in this study who had microalbuminuria was relatively small. However, the progression rates in the placebo group were again greater than those on treatment in both the microalbuminuric and normoalbuminuric groups. There was no real difference in risk of progression between the two treatment groups. Baseline albumin excretion rate therefore does not greatly influence the impact of lisinopril on retinopathy. All patients benefited from lisinopril treatment irrespective of their baseline albumin excretion.

\section{Action of ACE inhibitors}

Is the impact of ACE inhibitors on diabetic retinopathy in EUCLID a simple blood pressure lowering effect or do they have some other specific action?

The unadjusted odds ratio for risk of progression of retinopathy was halved in patients treated with lisinopril over a two year period. If adjustment is made for baseline covariates, such as treatment centre, blood glucose concentration, age, duration of diabetes, sex, baseline albumin excretion rate, baseline retinopathy status, and baseline blood pressure, the risk is attenuated to 0.55 . This means that the $50 \%$ reduction in risk is decreased to a $45 \%$ reduction in risk.

In the EUCLID study, blood pressure fell by about $3 \mathrm{~mm} \mathrm{Hg}$ diastolic at one month in the lisinopril group, and remained at that level throughout the study. If adjustment is now made for this difference in blood pressure the treatment effect is attenuated from 0.55 to 0.57 . This suggests that the systemic changes in blood pressure do not account for the treatment effect of ACE inhibitors on diabetic retinopathy.

This is supported by data on the progression of nephropathy from the EUCLID study. At two years, albumin excretion was 53\% lower with lisinopril compared to placebo. If blood pressure at one month is accounted for, this treatment effect is attenuated from $53 \%$ to $46 \%$. This is a small attenuation and further suggests that the effects of lisinopril on nephropathy are not caused by systemic blood pressure changes alone.

\section{Other mechanisms}

ACE inhibitors have a wide range of effects in addition to blood pressure lowering. For example, captopril can ameliorate arthritis and lower thrombotic risk. It reduces the risk of atherosclerosis and renal failure, and in some studies reduces the risk of cancer. All of these effects are, to some extent, dependent on angiogenesis. Importantly, angiogenesis is one of the key factors in the pathology of diabetic retinopathy.

ACE inhibitors are also known to have an effect on endothelial dysfunction. It is clear that people with type 1 diabetes have impaired endothelial dysfunction. If the ACE inhibitor 
enalapril is added, endothelial dysfunction is improved perhaps via mechanisms associated with nitric oxide bioactivity.

Interestingly, matrix metalloproteinases may also play a role. In an in vitro study showing degradation caused by matrix metalloproteinases, the degradation was attenuated when captopril was added. ${ }^{6}$

Furthermore, there are now studies showing that matrix metalloproteinase-9 (MMP-9) is increased approximately three months before the onset of microalbuminuria and that ACE inhibitors beneficially affect the level of MMP-9 and reduce the risk of microalbuminuria. ${ }^{7}$

ANGIOTENSIN II ANTAGONISTS

In a study of hypertensive patients, rather than diabetic patients, comparing losartan and enalapril, there is a similar effect on albumin excretion rate at 12 weeks. ${ }^{8}$ However, this should be interpreted with caution as the number of patients was small and the study was not designed to compare the impact of these two drugs.

\section{Conclusion}

Antihypertensive treatment, most notably with ACE inhibitors, reduces progression of diabetic retinopathy. There is evidence that ACE inhibitors may even reduce the incidence or development of retinopathy. Importantly, changes in systemic blood pressure cannot wholly account for the effect of ACE inhibitors on diabetic retinopathy.

In the future the findings of EUCLID and UKPDS, in terms of progression of retinopathy

\section{Trial acronyms}

DCCT: Diabetes Control and

Complications Trial

EUCLID: EUrodiab Controlled trial of Lisinopril in Insulin dependent Diabetes mellitus

UKPDS: United Kingdom Prospective Diabetes Study

and incidence, need to be confirmed in both type 1 and type 2 diabetes. Attention must also be paid to the more specific blockade of angiotensin II with agents such as the $\mathrm{AT}_{1}$ receptor antagonists.

1 The Diabetes Control and Complications Trial Research Group. The effect of intensive treatment of diabetes on the development and progression of long-term complications in insulin-dependent diabetes mellitus. $N$ Engl $f \mathrm{Med}$ in insulin-dependent

2 Volpert OV, Ward WF, Lingen MW, et al. Captopril inhibits angiogenesis and slows the growth of experimental tumors angiogenesis and slows the growth of

3 Parving HH, Larsen M, Hommel E, et al Effect of antihypertensive treatment on blood-retinal barrier permeability to fluorescein in hypertensive type 1 (insulindependent) diabetic patients with background retinopathy. Diabetologia. 1989;32:440-4.

4 UK Prospective Diabetes Study Group. Efficacy of atenolol and captopril in reducing risk of macrovascular and micro-
vascular complications in type 2 diabetes: UKPDS 39. BMJ 1998;317:713-20.

5 The EUCLID study group. Randomised placebo-controlled trial of lisinopril in normotensive patients with insulindependent diabetes and normoalbuminuria or microalbuminuria. Lancet 1997;349:1787-92.

6 Sorbi D, Fadly M, Hicks R, et al. Captopril inhibits the 72 $\mathrm{kDa}$ and $92 \mathrm{kDa}$ matrix metalloproteinases. Kidney Int 1993;44:1266-72.

7 Ebihara I, Nakamura T, Shimada N, et al. Increased plasma metalloproteinase-9 concentrations precede development of microalbuminuria in non-insulin-dependent diabetes mellitus. Am f Kidney Dis 1998;32:544-50.

8 Nielsen S, Dollerup J, Nielsen B, et al. Losartan reduces albuminuria in patients with essential hypertension. An enalapril controlled 3 months study. Nephrol Dial Transplant. 1997;12(suppl 2):19-23. 